\title{
Role of Alfvén Instabilities in Energetic Ion Transport
}

\author{
S. Bernabei, M.G. Bell, R. Budny, D. Darrow, E.D. Fredrickson, N. Gorelenkov*, J. C. Hosea, \\ R. Majeski, E. Mazzucato, R. Nazikian, C. K. Phillips, J.H. Rogers ${ }^{* *}$, G. Schilling, R. White, \\ J.R. Wilson, F. Zonca ${ }^{* * *}$, and S. Zweben
}

Princeton Plasma Physics Laboratory, P.O. Box 451, Princeton N.J. 08543

\begin{abstract}
Experiments of plasma heating at the ion cyclotron resonance of a minority specie have shown that the heating efficiency degrades above a certain power threshold. It is found that this threshold is due to the destabilization of a branch of shear Alfvén waves which causes a diffusive loss of fast ions, the Energetic Particle Modes. These modes not only play a fundamental role in the transport of the fast ions, but appear closely related to the formation of the giant sawteeth.

PACS \# $\quad 52.50 . \mathrm{Gj} \quad 52.55 . \mathrm{Fa} \quad 52.35 . \mathrm{Bj} \quad 52.30 . \mathrm{Jb}$
\end{abstract}

\section{INTRODUCTION}

Heating of tokamak plasmas by waves in the Ion Cyclotron Range of Frequencies (ICRF) often employs the minority-ion fundamental resonance coupling scheme[1]. This scheme creates an energetic population of ions of the minority species which equilibrates with the bulk ions and electrons through collisions. During such heating, the normal sawtooth relaxation instabilities are often suppressed. It has been postulated that the pressure exerted by the fast ion distribution created by ICRF minority heating, stabilizes the $m=1, n=1$ oscillation and therefore eliminates the sawteeth. A concurrent effect of the fast ion tail is the excitation of Alfvén instabilities which can cause scattering and, eventual, losses of fast particles. Several modes, different in structure and with a variety of toroidal numbers can be destabilized. They affect the transport of fast particles and therefore also play a fundamental role in the sawtooth stabilization and the eventual giant crash[2]. 
Experimental conditions and heating results are presented in section 2 and 3. Section 4 describes the kinds of Alfvén instabilities which are generated by the tail ions and their role in the transport of the fast ions. In section 5, the correlation between a particular kind of Alfvén instability and the formation and evolution of the giant sawtooth is described. Conclusions are in section 6 .

\section{EXPERIMENTAL CONDITIONS}

ICRF minority heating experiments were performed in a variety of plasma conditions in TFTR[3], with the purpose of heating and current drive: in almost all cases, a lengthening of the sawteeth was obtained. The experiments were performed in deuterium or helium-4 plasmas with ICRF heating at the fundamental minority hydrogen resonance. Frequencies 43, 47 and 63.6 $\mathrm{MHz}$ were used with corresponding magnetic fields to produce the cyclotron resonance on axis. The average density ranged between 2 and $4.6 \times 10^{19} \mathrm{~m}^{-3}$. The plasma current varied between 1.2 and 1.8 MA, in such a manner that $\mathrm{q}(\mathrm{a}) \approx 3.2$ in the great majority of cases. The MHD activity was monitored by means of a set of Mirnov coils[4], which consisted of a poloidal array of coils to determine the poloidal mode number m, and a toroidal array of coils to determine the toroidal mode number $n$. These measurements were supplemented by data from a microwave reflectometer[5] whenever available. The reflectometer gave information about density fluctuations caused by MHD modes in the core of the plasma. Energetic ions ejected from the plasma as a result of their interaction with the MHD activity were monitored by means of four probes placed at the edge of the plasma [6].

\section{ICRF MINORITY HEATING}

During ICRF minority heating, the rf power is preferentially coupled to the minority species, creating an energetic tail in the distribution function for sufficiently high input power. At low power, the minority ions are quickly thermalized by collisions with the ions, and the tail contains very little total energy. At higher power minority ions thermalize through collisions with electrons and a fast ion tail begins to form. It is approximately at this power level that Alfvén instabilities appear, and, since they cause losses, the rate of increase of the stored energy is smaller. Figure 1 shows that the stored energy increases linearly with power 
up to $\approx 3 \mathrm{MW}$. Above this power the rate of increase of the energy is lower. The peak electron temperature presents a break in slope at the same power level and at this power level, the probes placed at the edge of the plasma register an increased flux of fast particles while the Mirnov loops detect activity in the Alfvén range of frequencies $(\sim 150-250 \mathrm{kHz})$. The intensity of the signal from the lost ion detectors is closely correlated with the amplitude of the Alfvén instabilities.

\section{ALFVÉN INSTABILITIES}

There are typically two distinct groups of modes detected in the Alfvén range of frequencies, one which displays the characteristic density $\left(n_{e}\right)^{-1 / 2}$ dependence of the Toroidal Alfvén Eigenmodes (TAE)[7], the other characterized by a chirping frequency[8] (Figure 2). Both groups are usually composed of several modes with well defined toroidal numbers. The TAE are global modes that are readily detected by the Mirnov coils. The chirping modes are detected as density fluctuations in the core by a reflectometer[8], before appearing in the Mirnov loop signals: this suggests that the frequency decrease is accompanied by an outward radial movement of the modes.

Always accompaning these instabilities there are burst modes at $\sim 15 \mathrm{kHz}$, which have $\mathrm{n}=1$, and are very similar to fishbones (Figure 3 ). The precise role of these modes has yet to be established, but they do not appear correlated to major ion losses.

The Nova-K code [9] does not calculate instability for any mode with characteristics of the chirping modes which are in the Alfvén continuum. On the other hand the HINST code [10] has calculated a very strong growth rate for the Energetic Particle Mode (EPM) $[11,12]$ (Figure 4), which appears to match the observed characteristics of the chirping modes. The EPMs can be destabilized by the ICRF, which creates a very energetic and very peaked fast ion distribution in the core.

The EPMs rather than the TAEs appear to be responsible for most of the fast ion losses. Figure 5 shows the correlation between the onset of the EPMs, the fast ion losses and the "clamping" of the total stored energy. At $t=3.8$ the fast ion losses increase sharply, the stored energy, which was recovering from the previous giant sawtooth, stops increasing and the spectrum of the TAEs is modified. It is at this time that the EPMs are destabilized in the 
core, but are detected by the Mirnov loops after a delay of $\sim 30 \mathrm{msec}$ since, being core localized modes, they have to propagate far enough outward to be detected at the edge. During the time between the giant sawtooth and the appearance of the EPMs, the TAEs are present, but they do not prevent the stored energy from increasing. The EPMs are destabilized first, even if they are predicted to require a stronger drive than the TAEs [13](Figure 6). It appears that the TAEs are destabilized by the flow of fast particles which cross the TAE location, displaced either by a giant sawtooth (Figure 7) or by the EPMs. Figure 8 shows the radial distribution of the fast ion beta calculated by TRANSP[14]: the fast ion distribution is created by ICRF well localized in the core with a very steep gradient and does not extend to the TAEs location. Without EPMs, TRANSP predicts a linear increase of the stored energy (Figure 9).

An indirect proof of the expulsion of fast ions from the core can be gained by measuring the difference in frequency of the various harmonics of the TAE. This difference is due to Doppler shift caused by the toroidal rotation of the plasma, with the frequency defined by $\quad \omega_{\text {exp }}=\omega_{\text {real }}-\mathrm{n} \omega_{\text {rot }}$ where $\omega_{\text {exp }}$ is the measured frequency, $\omega_{\text {rot }}$ the rotation frequency and $\omega_{\text {real }}$ the real frequency. Before the appearance of the EPMs the rotation is calculated to be $\sim 28 \mathrm{~km} / \mathrm{sec}$, in very good agreement with the spectroscopically measured value of $30 \mathrm{~km} / \mathrm{sec}$. During the EPMs activity, it drops to $\sim 22 \mathrm{~km} / \mathrm{sec}$. The counter-rotation is provided by the force $\mathrm{E}_{\mathrm{r}} \mathrm{xB}$, where $\mathrm{Er}$ is the electric field induced by the expulsion of ions from the core (Figure 10).

Initial modelling has been done with the ORBIT code [15]. This code evolves a fast ion distribution generated by TRANSP modelling of the ICRF heating. This modelling can give only a qualitative description of the processes leading to fast ion losses, since some essential quantities such as amplitude are unmeasurable.

The results can be summarized as follows. Necessary conditions for ion transport and eventual losses are: 1) there must be a local resonant wave-particle interaction; 2) the modes must have significant amplitude; 3) the modes must have broad radial extent or be radially sweeping. The TAEs are stationary, global modes, but being heavily damped in the core where the fast ions are created, do not contribute to significant ion losses. The EPMs on the other hand, by sweeping a portion of the radius can produce a large diffusive loss of fast ions 
$[16,17]$. This is seen in Figure 11. The increase of the perpendicular energy in the ICRF tail ions is clamped during the chirping of the frequency of the EPMs, and recovers when the frequency locks into a stationary value.

\section{ROLE OF ALFVÉN INSTABILITIES IN GIANT SAWTEETH}

Upon application of ICRF power to the plasma the sawtooth period lengthens as does the height of the crash. It is believed that the fast ions contribute a rigidity to the plasma, thereby making the $\mathrm{m}=1$ oscillations more difficult[18].

In the same discharge there can be two kinds of giant sawteeth, one with a period up to 3 times longer than the ohmic sawtooth and one which is about 20 times longer $(\sim 600-700$ msec) (Figure 12). It is interesting to note that the long sawteeth all have EPM activity, the shorter do not. It is also interesting to note that there are no intermediate values of the period, as if a kind of bifurcation existed.

There is the possibility that EPMs can develop as a result of the long sawtooth. But all the giant sawteeth present an interesting characteristic which seems to imply that the EPMs play a fundamental role in their dynamics. The sawtooth crash does not occur at the maximum of the EPMs' activity, and therefore at the maximun of the ion losses, but only after the chirping of the EPMs frequency almost stops and the frequency itself locks (see fig. 11).

It appears that somehow the action of the EPMs contributes to delay the inward diffusion of the flux: several mechanism are being investigated.

\section{CONCLUSIONS}

Experiments have been conducted in TFTR employing the ion cyclotron resonance of a minority species on the axis of the plasma in dischrages at low q(a). Under these conditions, the ICRF power creates a very energetic population of fast ions in the core of the plasma. Above a certain threshold, this energetic tail drives Energetic Particle Modes unstable. The EPMs, characterized by a chirping frequency, cause radial displacement of the energetic particles which leads to a diffusive loss of the particles themselves. The heating efficiency results therefore degraded. 
Giant sawteeth, characteristic of these low q(a) discharges, are always accompanied by EPMs and a close correlation is found between the two.

The phenomenology of the ICRF fast ions in the core has several similarities with alpha particles, therefore modelling for reacting plasmas should take into account the EPMs. 


\section{Figure Captions}

Figure 1. Correlation between stored energy, integrated mode amplitude and lost ions vs. rf power. Conditions were: plasma current 1.68 MA, toroidal field 3.0 T, average density $2.8 \times 10^{19} \mathrm{~m}^{-3}$ and RF frequency $43 \mathrm{MHz}$.

Figure 2. Frequency spectrum of the Alfvén instabilities from a Mirnov loop; the numbers refer to the toroidal wavenumber of the modes. TAEs are seen at approximately constant frequency between 210 and $220 \mathrm{kHz}$, while the EPMs sweep in frequency.

Figure 3. Mirnov loop frequency spectrum of the fishbone-like burst modes in the same discharge as shown in figure 2.

Figure 4. Frequency $(\omega)$ and growth rate $(\gamma)$ of the EPM calculated by HINST code; $\mathrm{P}_{\mathrm{rf}}=6.3 \mathrm{MW}$.

Figure 5. Correlation between EPM excitation, lost ions and stored energy: losses increase and stored energy saturates at the onset of the EPMs. Conditions as in figure 1, $\mathrm{P}_{\mathrm{rf}}=6.3 \mathrm{MW}$

Figure 6. Mirnov loop frequency spectra for three conditions with increasing rf power. note that the duration of the activity of the EPMs before the giant sawtooth is practically independent of rf power. The EPMs, in all cases, appear before the onset of TAEs.

Figure 7. TAEs bursts due to fast ions displaced by the sawtooth crashes.

Figure 8. Radial density profile of fast ions as computed by TRANSP, before sawtooth mixing. There is essentially no fast ion density at the radius of the TAEs and hence they are stable until a sawtooth crash occurs. Conditions as in figure 5.

Figure 9. Comparison of the measured and TRANSP-computed stored energy for the conditions of figure 5. The stored energy falls below that computed when the EPMs begin, indicating that they are primarily responsible for the losses of the fast ions.

Figure 10. Decrease of toroidal rotation during EPMs activity.

Figure 11. The evolution of the perpendicular energy stored in the tail ions is clamped during the chirping of the EPMs, and recovers when their frequency locks. The 
EPM's amplitude is multiplied by the variation of frequency as a measure of the chirping. Maximum fast ion losses occur at the time when sweeping is strongest.

Figure 12. Sawtooth period for the experimental conditions of figure 1. Open circles without EPMs, full circles with EPMs. Note the absence of intermediate periods between the two situations. 


\section{Acknowledgements}

We gratefully acknowledge very enlightening discussions with Professor L. Chen and Dr. T. Hoang. Work supported by the U.S. Department of Energy under contract No. DEAC02-76-CH0-3073.

\section{References}

[1] J. R. Wilson et al., in Plasma Physics and Controlled Nuclear Fusion Research 1992 (Proc. 14th Int. Conf. Würzburg, 1992), vol.1 (IAEA, Vienna 1993) 661.

[2] R. B. White et al., Phys. Plasmas 2, (1995) 2871.

[3] R. Hawryluk et al., Plasma Phys. Controlled Fusion 33, (1991) 1509.

[4] E. D. Fredrickson et al., Rev. Sci. Instrum. 66, (1995) 813.

[5] E. Mazzucato and R. Nazikian, Phys. Rev. Lett. 71, (1993) 1840.

[6] D. Darrow, S. J. Zweben, Z. Chang et al., Nucl. Fus. 37, (1997) 939.

[7] K. L. Wong et al., Plasma Phys. Control. Fusion 36, (1994) 879.

[8] E. D. Fredrickson et al., Nucl. Fus. 35, (1995) 1457.

[9] G. Y. Fu, C. Z. Cheng, R. Budny et al., Phys. Plasmas 3, (1996) 4036.

[10] N. N. Gorelenkov et al. Phys.Plasmas 5, (1998) 3389.

[11] F. Zonca and L. Chen, Phys. Plasmas 3, (1996) 323.

[12] C. Z. Cheng, N. N. Gorelenkov, C. T. Hsu, Nucl. Fus. 35, (1995) 1639.

[13] S. Briguglio et al., in Plasma Physics and Controlled Nuclear Fusion Research (Proc. 16th Int. Conf. Montreal, 1996), vol.2 (IAEA, Vienna 1997) 543.

[14] R. V. Budny et al., Nucl. Fus. 35, (1995) 1497.

[15] R. B. White and M. S. Chance, Phys. Fluids 27, (1984) 2455.

[16] H. E. Mynik and N. Pomphrey, Nucl. Fus. 34, (1994) 1277.

[17] C. T. Hsu, C. Z. Cheng, P. Helander et al., Phys. Rev. Lett. 72, (1994) 2503.

[18] B. Coppi et al., Phys. Rev. Lett. 63, (1989) 2733.

\footnotetext{
* TRINITI, Troitsk, Moscow, Russia 142092

** Intevac Inc., 3550 Basset St., Santa Clara, Ca. 95054

**** Associazione EURATOM-ENEA, CRE 00044 Frascati, Italy
} 


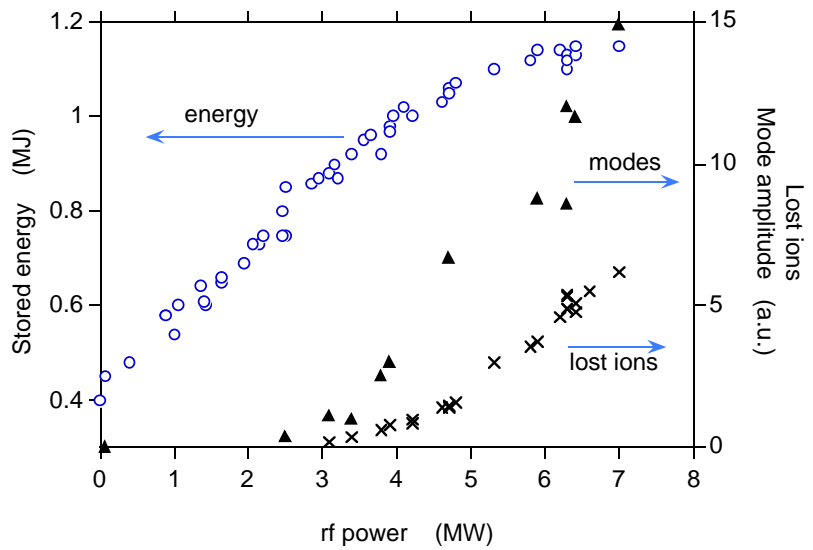

Figure 1. Correlation between stored energy, integrated mode amplitude and lost ions vs. If power. Conditions were: plasma current 1.68 MA, toroidal field 3.0 T, average density $2.8 \times 10^{19} \mathrm{~m}^{-3}$ and rf frequency $43 \mathrm{MHz}$. 


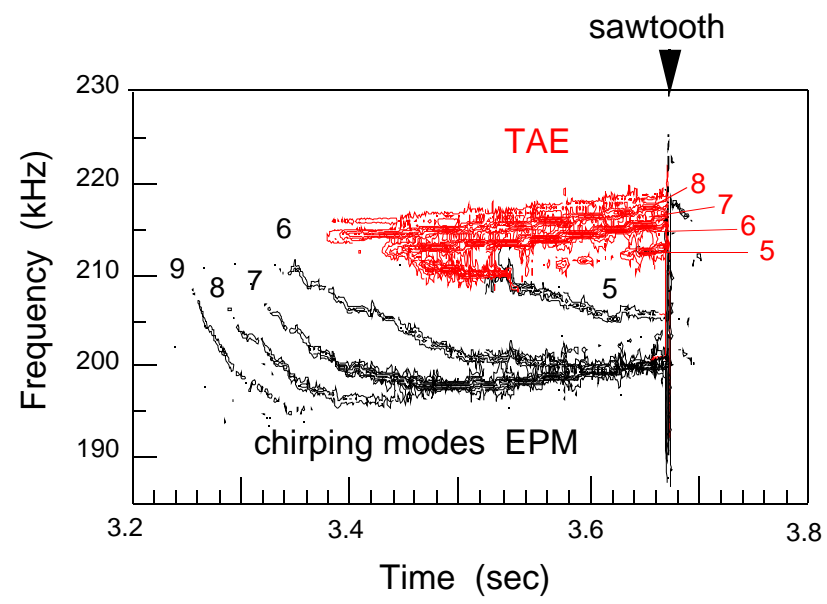

Figure 2. Frequency spectrum of the Alfvén instabilities from a Mirnov loop; the numbers refer to the toroidal wavenumber of the modes. TAEs are seen at approximately constant frequency between 210 and $220 \mathrm{kHz}$, while the EPMs sweep in frequency. 


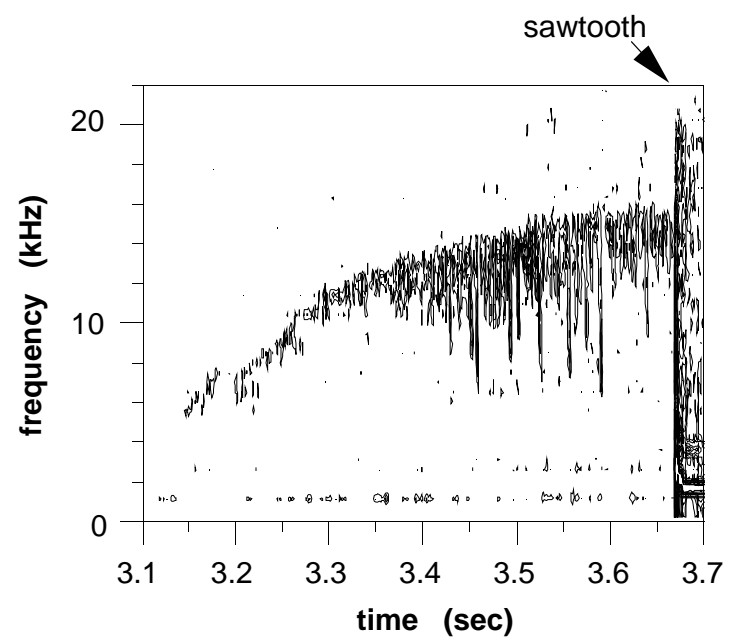

Figure 3. Mirnov loop frequency spectrum of the fishbone-like burst modes in the same discharge as shown in figure 2. 


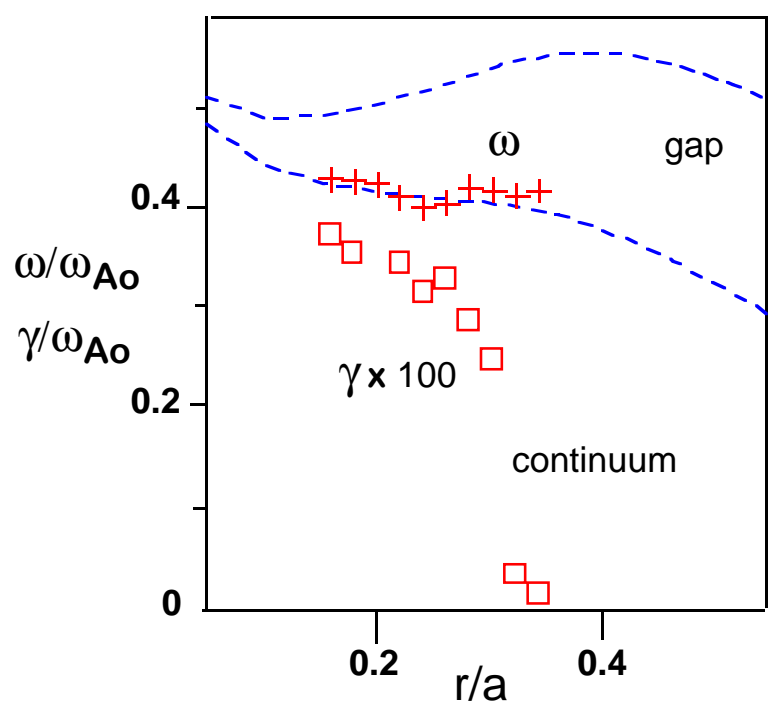

Figure 4. Frequency ( $\omega)$ and growth rate $(\gamma)$ of the EPM calculated by HINST code; $P$ rf $=6.3 \mathrm{MW}$. 


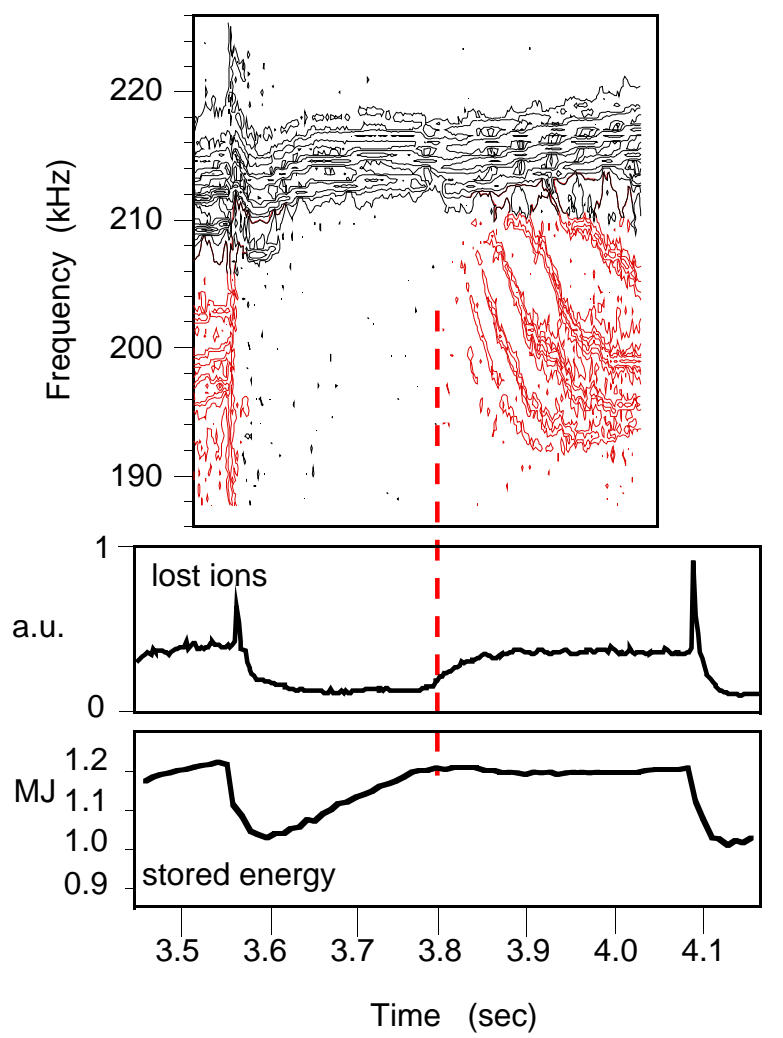

Figure 5. Correlation between EPM excitation, lost ions and stored energy: losses increase and stored energy saturates at the onset of the EPMs. Conditions as in figure $1, \mathrm{P}_{\mathrm{rf}}=6.3 \mathrm{MW}$ 


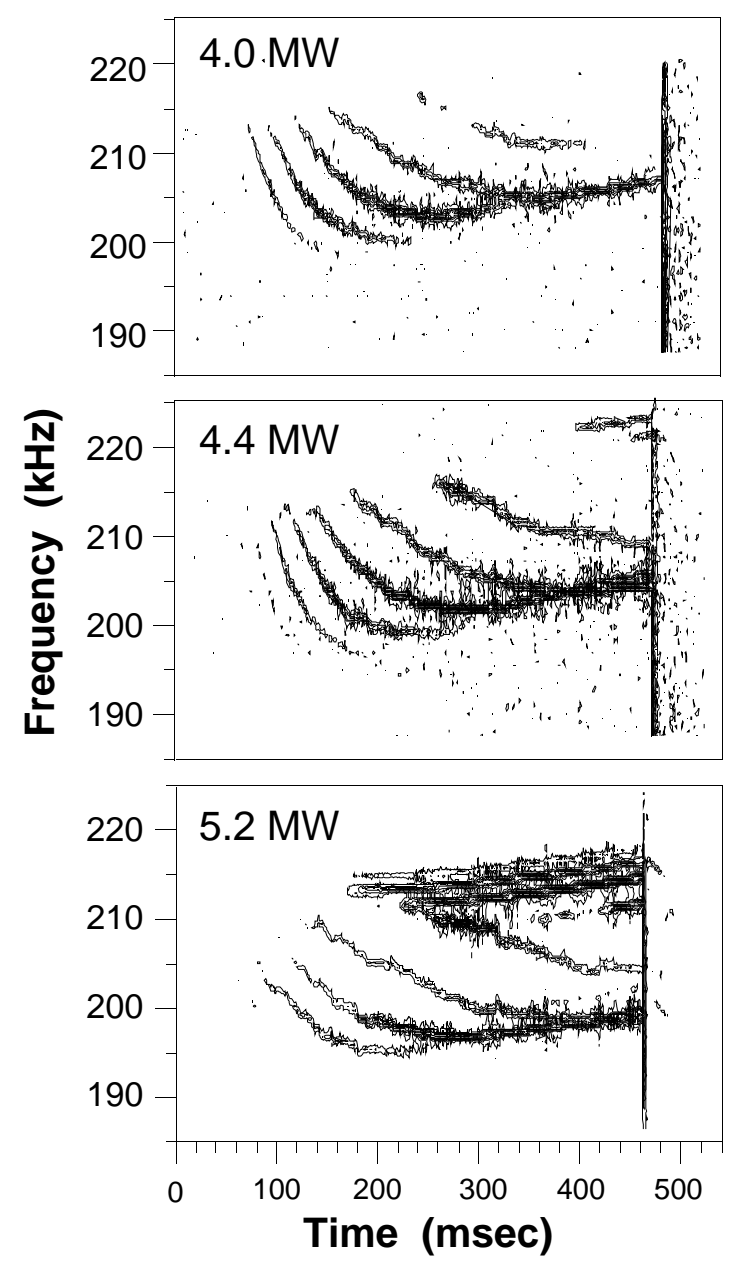

Figure 6. Mirnov loop frequency spectra for three conditions with increasing rf power. Note that the duration of the activity of the EPMs before the giant sawtooth is practically independent of rf power. The EPMs, in all cases, appear before the onset of TAEs. 


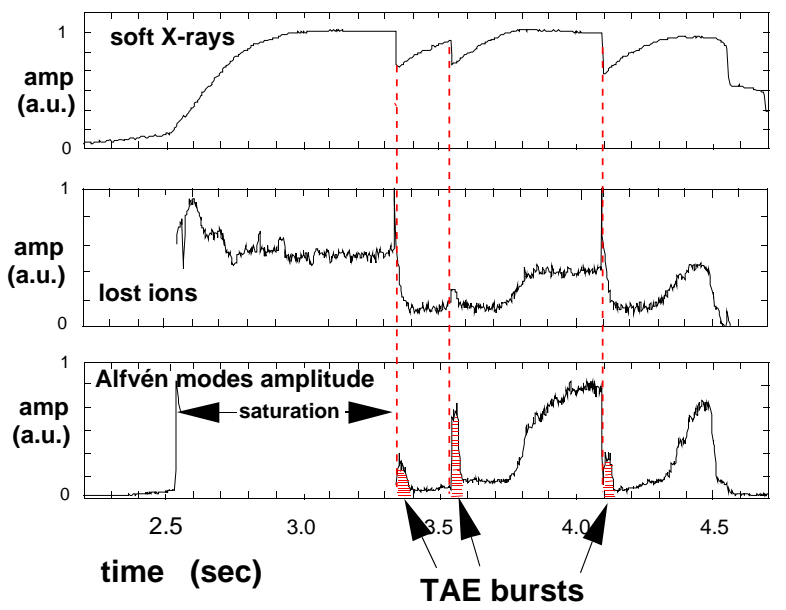

Figure 7. TAEs bursts due to fast ions displaced by the sawtooth crashes. 


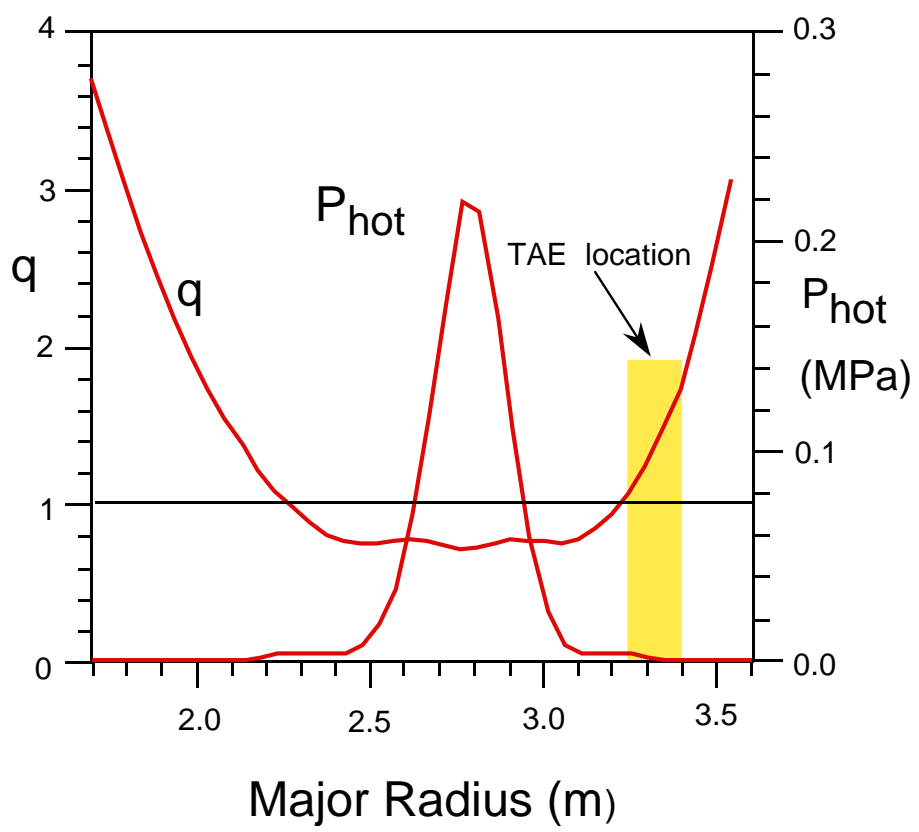

Figure 8 . Radial density profile of fast ions as computed by TRANSP, before sawtooth mixing. There is essentially no fast ion density at the radius of the TAEs and hence they are stable until a sawtooth crash occurs. Conditions as in figure 5 . 


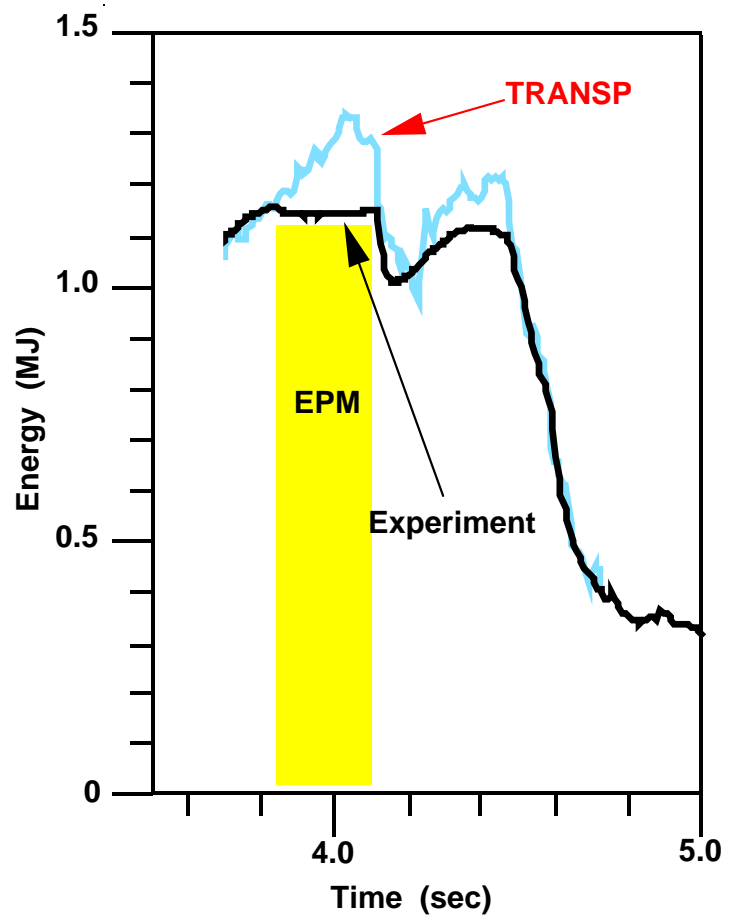

Figure 9. Comparison of the measured and TRANSP-

computed stored energy for the conditions of figure 5 . The stored energy falls below that computed when the EPMs begin, indicating that they are primarily responsible for the losses of the fast ions. 


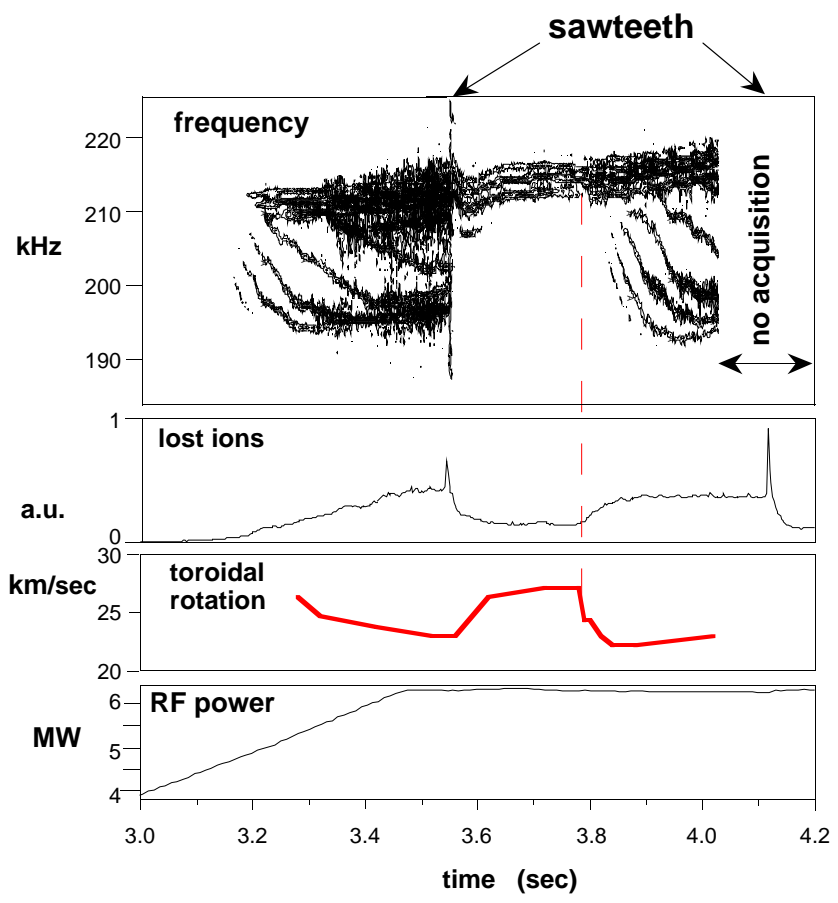

Figure 10. Decrease of toroidal rotation during EPMs activity. 


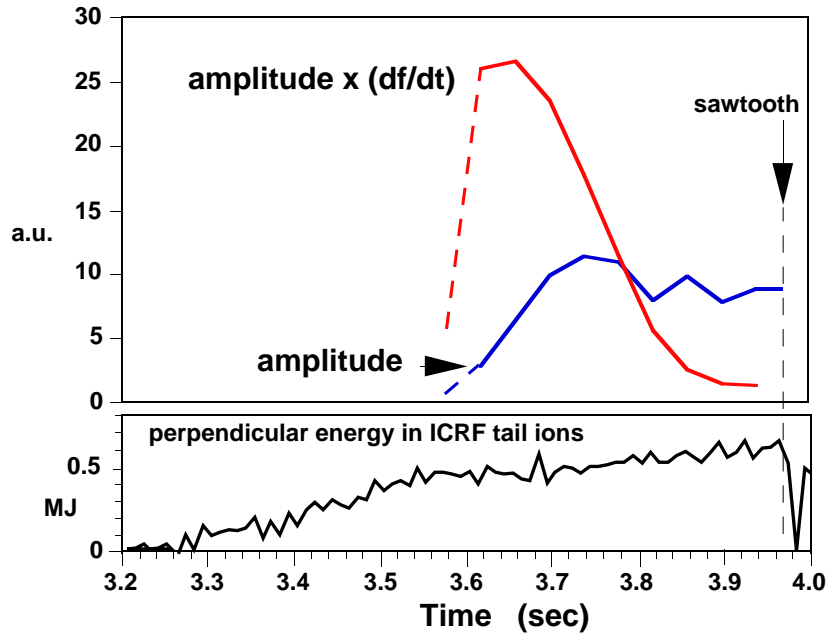

Figure 11. The evolution of the perpendicular energy stored in the tail ions is clamped during the chirping of the EPMs, and recovers when their frequency locks. The EPM's amplitude is multiplied by the variation of frequency as a measure of the chirping. Maximum fast ion losses occur at the time when sweeping is strongest. 


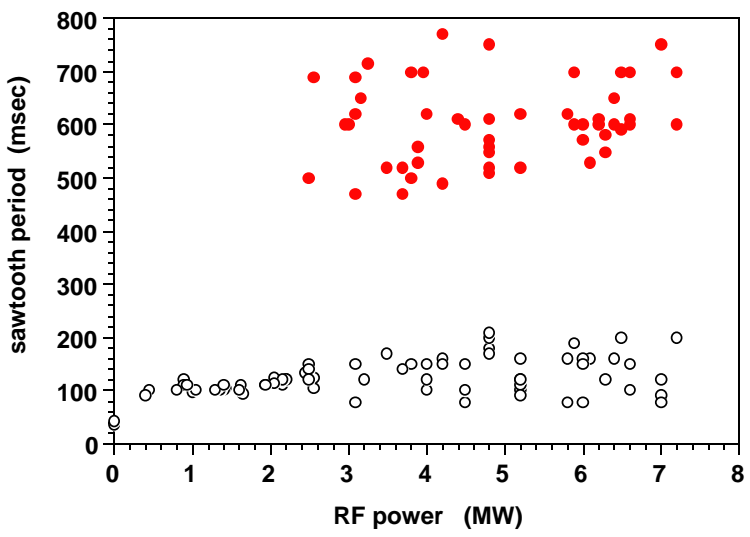

Figure 12. Sawtooth period for the experimental conditions of figure 1. Open circles without EPMs, full circles with EPMs. Note the absence of intermediate periods between the two situations. 